\title{
Étudiants en médecine et Internet : quelles pratiques de recherche et comment les améliorer?
}

\section{Medical students and the Internet: What are their research practices and how can they be improved?}

Carine ZUMSTEIN ${ }^{1, *}$, Claire KAM ${ }^{1,2}$, Pierrick Le BORGNE ${ }^{1,2}$, François LEFEBVRE ${ }^{3}$, Michel LEVEQUE ${ }^{4}$, et Pierre VIDAILHET ${ }^{1,5}$

${ }^{1}$ Unité de simulation européenne en santé (UNISIMES), Faculté de médecine, Université de Strasbourg, 4 rue Kirschleger, 67000 Strasbourg, France

2 Service d'accueil des urgences adultes, Hôpitaux universitaires de Strasbourg, Hôpital de Hautepierre, 1 avenue Molière, 67200 Strasbourg, France

3 Département de santé publique, Hôpitaux universitaires de Strasbourg, 1 place de l'Hôpital, 67000 Strasbourg, France

${ }^{4}$ Département de médecine générale, Faculté de médecine, Université de Strasbourg, 4 rue Kirschleger, 67000 Strasbourg, France

${ }^{5}$ Pôle de psychiatrie, de santé mentale et d'addictologie, Hôpitaux universitaires de Strasbourg, Strasbourg, France

Manuscrit soumis le 4 avril 2017 ; commentaires éditoriaux formulés aux auteurs le 9 novembre 2017 et le 27 mars 2018 ; accepté pour publication le 31 mars

Résumé-Introduction : Internet est un outil utile aux médecins pour compléter leurs connaissances. Le but de cette étude était d'identifier les pratiques de recherche sur Internet des étudiants en médecine et d'évaluer leur amélioration par une action de sensibilisation. Méthodes : Quatre-vingt-dix étudiants de la faculté de médecine de Strasbourg ont été randomisés en deux groupes. Le groupe A a répondu à des questions annexées à un cas clinique, puis à un questionnaire déclaratif concernant leurs pratiques habituelles de recherche d'informations médicales sur Internet. Le groupe B a d'abord été sensibilisé à ces pratiques grâce au questionnaire avant de répondre au cas clinique. Notre analyse a porté sur l'historique de leurs recherches, les scores obtenus par les étudiants et sur l'auto-évaluation de leurs pratiques. Résultats: Les étudiants déclarent consulter indifféremment des sites médicaux de référence et des sites de vulgarisation médicale, résultats retrouvés expérimentalement avec le cas clinique. Une action de sensibilisation préalable favorise l'utilisation de Google.fr et la consultation de sites de référence comme celui de la Haute Autorité de santé, et réduit la consultation de sites de vulgarisation. La sensibilisation incite les étudiants à multiplier leurs sources d'information. Les étudiants ayant utilisé Internet ont obtenu de meilleures notes que ceux ayant répondu à l'aide de leurs seules connaissances. Conclusion : Internet permet de compléter efficacement les connaissances médicales. Les étudiants sensibilisés à leurs pratiques de recherche sur Internet consultent des sites de meilleure qualité tout en multipliant leurs sources d'information. Il est nécessaire de développer de meilleures formations à l'utilisation de cet outil.

Mots clés : internet, recherche documentaire, étudiants en médecine, formation médicale

\begin{abstract}
Background: The Internet is a useful but often misused tool for physicians to improve their medical knowledge. We conducted this study to observe medical students' research practices on the Internet and to evaluate their improvement by raising their awareness using a questionnaire. Methods: Ninety students from the University of Strasbourg were randomised in two groups. The first group answered clinical questions then filled out a questionnaire about their professional use of the Internet. The second group was educated about their research practices on the Internet with the questionnaire before they answered the clinical questions. We analysed their web search history, responses to the clinical questions, and the answers given on the questionnaire. Results: Students declared that they consulted indistinctly medical reference websites and
\end{abstract}

\footnotetext{
*Correspondance et offprints: Carine ZUMSTEIN, Unité de simulation européenne en santé (UNISIMES). Faculté de médecine, Université de Strasbourg, 4 rue Kirschleger, 67000 Strasbourg, France.

Mailto: carine.zumstein@gmail.com.
} 


\begin{abstract}
popular science websites. Using an awareness questionnaire significantly increased their use of Google.fr and reference sites such as the HAS website (French National Authority for Health) when responding to medical questions. We observed a tendency to consult the WHO website more often, and to consult popular science websites, such as Wikipedia.org and Doctissimo.fr, less often. Students in the second group enriched their information sources when responding to clinical questions. Students who used the Internet obtained higher marks compared to those who answered with their own knowledge. Conclusion: The Internet allows students to complement effectively their medical knowledge. Students aware of their search practices consult more reliable websites, while enriching their medical information resources. We need to develop appropriate methods to train medical students regarding their Internet use.
\end{abstract}

Keywords: internet, literature search, medical students, medical education

\section{Introduction}

L'évolution constante des technologies informatiques permet aujourd'hui à chacun un vaste accès aux avancées de la recherche scientifique et médicale via Internet [1-3]. Les professionnels de la santé peuvent ainsi s'appuyer sur Internet pour compléter leurs connaissances, en s'appuyant, par exemple, sur les dernières mises à jour des recommandations de bonnes pratiques de soins. Cependant, Internet est un réseau d'information provenant de sources multiples avec des références plus ou moins identifiables. La recherche sur Internet est, par ailleurs, complexifiée par le choix des mots-clés et des moteurs de recherche qui ne distinguent pas les sites destinés aux professionnels des médias à usage «grand public ». Les informations pertinentes et validées sont donc difficiles à identifier et leur fiabilité reste incertaine, vu le manque fréquent de données concernant leurs auteurs et leurs qualifications.

Savoir rechercher sur Internet des informations fiables relève de la capacité de l'individu à solliciter des ressources externes et à adopter une posture réflexive vis-à-vis de ses pratiques de recherche. Les médecins reconnaissent à ce propos avoir besoin de meilleures connaissances et de méthodes en matière de recherche d'informations médicales sur Internet, comme l'ont montré les travaux de Ricard et al. [4] et Hattab et al. [5] au moyen de questionnaires déclaratifs auprès d'étudiants du deuxième cycle des études médicales en France. Comme le montrent plusieurs autres études [1-3,6-8], les médecins s'interrogent sur leurs pratiques, car elles peuvent avoir des conséquences sur la qualité de la prise en charge de leurs patients. Fournir des informations pertinentes aux patients concernant leurs diagnostics et leurs traitements est important pour favoriser leur participation aux décisions médicales et leur adhésion aux soins.

D'autre part, dans la mesure où les patients consultent régulièrement Internet pour des questions relatives à leur santé et pour examiner de façon critique les dires de leurs médecins [1,2,6-10], la capacité de ces derniers à les guider dans leurs choix pourrait aider à établir une relation de confiance [11]. Or, dans l'étude de Ricard et al. [4], les sites que les étudiants déclaraient consulter pour leur formation étaient d'abord des sites créés et financés par l'industrie pharmaceutique; lors d'une mise en situation de recherche d'un document pour préparer leurs examens, les 10 étudiants testés n'ont pas consulté les sites institutionnels qui leur sont destinés. Dans un travail plus récent d'Egle et al. [9] utilisant un questionnaire en ligne, les étudiants en médecine déclaraient utiliser fréquemment les ressources Internet lorsqu'ils devaient apprendre rapidement ou prendre des décisions cliniques et thérapeutiques ; les sites principalement consultés étaient des sites «grand public», qui étaient jugés par les auteurs comme de faible qualité sur la base de la fiabilité des informations fournies. Utilisant différents moyens d'évaluation plus ou moins subjectifs, plusieurs études [1-3,5-8,12-14] confirment que de nombreux sites médicaux manquent de fiabilité. Eysenbach et al. [15] ont cherché à savoir comment les patients évaluent la pertinence des sites médicaux; au travers d'une recherche qualitative basée sur des groupes de discussion focalisée et une mise en situation, leurs résultats montrent que, malgré leurs affirmations, les patients n'ont pas cherché à évaluer la fiabilité des sites consultés.

L'objectif de notre étude était d'étudier les pratiques de recherche sur Internet des étudiants en médecine sur la base d'un questionnaire déclaratif, mais aussi à partir d'une mise en situation simulée consistant à rechercher des informations médicales pour répondre rapidement à des questions cliniques et d'évaluer leur possibilité d'améliorer leurs pratiques de recherche par une action de sensibilisation.

\section{Méthodes \\ Population}

Nous avons recruté 90 étudiants inscrits en troisième année de diplôme de formation approfondie en sciences médicales (DFASM3, c'est-à-dire en sixième et dernière année du cursus pré-gradué des études médicales) à la Faculté de médecine de Strasbourg (France) pour l'année universitaire 2014/2015 (Tableau I). Nous avons recruté les étudiants à l'Unité de simulation européenne en santé (UNISIMES) de Strasbourg à la fin d'une formation organisée en petits groupes sans aucun rapport avec l'étude. Il n'y avait aucun critère d'exclusion.

En dix minutes, nous avons expliqué à chaque groupe l'objectif de notre recherche qui était d'évaluer leur façon de répondre à des questions cliniques avec l'aide d'Internet grâce à un protocole d'étude d'environ 30 minutes comportant l'exposition à un cas clinique, introduisant sept questions qui exploraient des connaissances cliniques ou thérapeutiques, et l'administration d'un questionnaire 
Tableau I. Caractéristiques démographiques des groupes d'étudiants en médecine ( $n=90$ étudiants).

\begin{tabular}{llll}
\hline Description de la population étudiée & & Groupe A & Groupe B \\
\hline \multirow{2}{*}{ Áge (années) } & Moyenne & $24,5 \pm 1,9$ & $24,8 \pm 2,6$ \\
& Non connu & $2(4,8 \%)$ & $3(6,2 \%)$ \\
Sexe & Féminin & $24(57,1 \%)$ & $30(62,5 \%)$ \\
& Masculin & $17(40,5 \%)$ & $15(31,2 \%)$ \\
& Non connu & $1(2,4 \%)$ & $3(6,3 \%)$ \\
Niveau d'études médicales & DFASM3 primant & $40(95,2 \%)$ & $45(93,7 \%)$ \\
& DFASM3 redoublant & $1(2,4 \%)$ & $3(0 \%)$ \\
Total & Non connu & $1(2,4 \%)$ & $n=4)$ \\
\hline
\end{tabular}

Groupe A : débutant par le cas clinique, Groupe B : débutant par le questionnaire, DFASM3 : $3^{\text {e }}$ année de Diplôme de Formation Approfondie en Sciences Médicales.

en ligne, à documenter dans un ordre défini pour chaque groupe. Les étudiants ont pu poser toutes les questions qu'ils souhaitaient. Aucun d'eux n'a refusé de participer et chacun a donné son consentement individuel par écrit après avoir pris connaissance des informations contenues dans la feuille de consentement (Annexe 1). L'étude se déroulait juste après. Cette étude a été déclarée au registre des activités de traitements conformément aux dispositions réglementaires de la loi informatique et liberté et a obtenu l'avis favorable consultatif d'un comité d'éthique.

\section{Méthodes}

\section{Dispositifs de recueil des données}

Les étudiants devaient répondre à sept questions introduites par un cas clinique. Le cas clinique était composé d'un court énoncé suivi de sept questions de médecine générale (Annexe 2). Les questions étaient :

- Quel est le traitement des hémorrö̈des?

- Quels sont les symptômes d'un syndrome de sevrage tabagique?

- Quels en sont les traitements possibles?

- Quelle est la définition du syndrome de Diogène?

- Citez dix moyens de contraception

- Combien y a-t-il d'envenimations et de décès par morsure de serpent dans le monde chaque année?

- Quelles sont les recommandations à donner en cas de morsure par un serpent?

Le choix de ces thèmes visait à simuler une consultation dans laquelle le médecin ne connaît pas tous les éléments de réponse mais peut s'aider d'Internet pour actualiser ses connaissances et mieux répondre aux attentes de son patient.

Le questionnaire (Annexe 3) les interrogeait sur les sites couramment visités et leur pertinence dans un contexte de recherches sur Internet lors de consultations médicales. Le but de ce questionnaire était de les sensibiliser par une prise de conscience concernant la qualité des sites utilisés, une analyse réflexive concernant leurs pratiques, et de leur suggérer des moyens pour les améliorer sans donner directement des conseils de recherche. Le questionnaire se composait de questions démographiques (âge, sexe, année d'études), de questions concernant les sites Internet qu'ils avaient consultés dans les six derniers mois et la fiabilité qu'ils accordaient à ces sites. Des questions supplémentaires leur demandaient s'ils utilisaient leur environnement numérique de travail (ENT) pour accéder aux bases de données numériques de l'Université de Strasbourg, le nombre moyen de sites consultés pour une même question scientifique ou médicale, et s'ils connaissaient le logo HONcode (logo reconnu par la Haute Autorité de santé pour la certification des sites de santé).

Les étudiants avaient accès à un ordinateur avec connexion à Internet pour mener leurs recherches. Ils ont été randomisés en deux groupes. Le groupe A répondait d'abord au cas clinique, puis au questionnaire, alors que le groupe B était d'abord sensibilisé aux pratiques de recherche sur Internet au moyen du questionnaire avant de répondre au cas clinique.

\section{Collecte des données}

Les données ont été collectées grâce aux ordinateurs de l'UNISIMES. Nous avons installé le logiciel traqueur «about:me 0.7 » sur le navigateur Internet Mozilla Firefox qui était imposé aux étudiants. Le logiciel nous a permis de collecter les historiques de recherche de chaque étudiant après son passage. Nous avons collecté le nombre total d'Uniform Resource Locators (URL ou pages Internet Spécifiques) et de domaines (ou sites Internet) consultés ainsi que leurs noms, globalement et pour chaque question, et le temps total de l'épreuve dans chaque groupe. Nous avons aussi déterminé la fréquence des copier-coller, le pourcentage de participants n'ayant pas suivi les consignes, et pris en compte si les étudiants prenaient garde aux termes médicaux utilisés sans les confondre dans leurs réponses.

Le questionnaire nous a permis d'analyser les caractéristiques démographiques des étudiants, les sites Internet consultés dans les six derniers mois pour des questions de santé, la fiabilité accordée à chacun de ces sites, le nombre de sites consultés pour une unique question médicale et leur connaissance de l'ENT et du logo HONcode. 
Tableau II. Principaux sites Internet consultés dans les deux groupes d'étudiants lors de leur mise en situation (réponses aux questions médicales).

\begin{tabular}{|c|c|c|c|}
\hline $\begin{array}{l}\text { Pourcentage de } \\
\text { consultation des sites }\end{array}$ & $\begin{array}{l}\text { Groupe A } \\
(n=42)\end{array}$ & $\begin{array}{l}\text { Groupe B } \\
(n=48)\end{array}$ & $\begin{array}{l}\text { Degré de significativité } \\
\text { (test exact de Fisher) }\end{array}$ \\
\hline Google.fr & $90 \%$ & $100 \%$ & $p=0,04 ;$ IC $95 \%:[0,78$; infini], OR infini \\
\hline Wikipedia.org & $81 \%$ & $67 \%$ & $p=0,15 ; \mathrm{IC} 95 \%:[0,16 ; 1,37], \mathrm{OR}=0,47$ \\
\hline Planetoscope.com & $57 \%$ & $46 \%$ & $p=0,30 ; \mathrm{IC} 95 \%:[0,25 ; 1,58], \mathrm{OR}=0,64$ \\
\hline Doctissimo.fr & $31 \%$ & $19 \%$ & $p=0,22 ;$ IC $95 \%:[0,17 ; 1,52], \mathrm{OR}=0,52$ \\
\hline Ameli.fr ${ }^{*}$ & $29 \%$ & $38 \%$ & $p=0,50 ; \mathrm{IC} 95 \%:[0,57 ; 4,05], \mathrm{OR}=1,49$ \\
\hline Who.int ${ }^{*}$ & $21 \%$ & $35 \%$ & $p=0,17 ; \mathrm{IC} 95 \%:[0,71 ; 5,89], \mathrm{OR}=1,99$ \\
\hline Snfge.org ${ }^{*}$ & $19 \%$ & $27 \%$ & $p=0,46 ;$ IC $95 \%:[0,52 ; 4,97], \mathrm{OR}=1,57$ \\
\hline Has-sante.fr ${ }^{*}$ & $7 \%$ & $23 \%$ & $p=0,04 ; \mathrm{IC} 95 \%:[0,91 ; 22,95], \mathrm{OR}=3,81$ \\
\hline Yahoo.com & $7 \%$ & $4 \%$ & $p=0,66 ;$ IC $95 \%:[0,05 ; 5,23], \mathrm{OR}=0,57$ \\
\hline
\end{tabular}

Les résultats sont donnés en pourcentages des étudiants dans chaque groupe. Groupe A : débutant par le cas clinique, Groupe B: débutant par le questionnaire, WHO : World Health Organization (Organisation mondiale de la santé), SNFGE : Société nationale française de gastro-entérologie, HAS : Haute Autorité de santé.

* Désigne les sites considérés comme «références».

Les données ont été anonymisées et collectées par un des auteurs (CZ) qui a aussi coté les réponses au cas clinique suivant une grille de correction (Annexe 2) attribuant jusqu'à 10 points par question, sans double vérification. La grille de correction a été élaborée par le même auteur (CZ) sur la base d'une recherche sur Internet similaire à celle réalisée par les étudiants, afin de s'assurer qu'ils puissent avoir accès aux mots-clés leur rapportant des points. Les sites utilisés pour construire la grille de correction ont été choisis selon leur fiabilité estimée en fonction des auteurs, sources et date de rédaction.

\section{Analyse statistique}

L'analyse statistique descriptive des variables quantitatives s'est faite en donnant pour chaque variable les paramètres de position (moyenne, médiane, minimum, maximum, premier et troisième quartiles) ainsi que les paramètres de dispersion (variance, écart-type, étendue, écart interquartile). Le caractère gaussien des données a été testé par le test de Shapiro-Wilk. Le descriptif des variables qualitatives s'est fait en donnant les effectifs et proportions de chaque modalité dans l'échantillon. Chaque fois que cela a été utile, des tableaux croisés ont été donnés avec effectifs, proportions par ligne, proportions par colonne et proportions par rapport au total, pour chaque case du tableau.

L'analyse inférentielle pour les variables qualitatives s'est faite avec un test exact de Fisher. Pour chaque variable, le rapport de cotes et son intervalle de confiance à $95 \%$ ont été estimés avec la méthode du maximum de vraisemblance. Les comparaisons de variables quantitatives entre groupes ont été réalisées soit par un test de Student lorsque la variable d'intérêt était gaussienne, soit par un test non paramétrique dans le cas contraire (test de Mann-Whitney-Wilcoxon). Des modèles linéaires mixtes ont permis d'étudier l'influence de la consultation des sites Internet sur la note à chaque question. Les analyses ont été réalisées avec le logiciel $\mathrm{R}$ dans sa version 3.1.0 ainsi qu'avec tous les progiciels requis pour mener à bien les analyses.

\section{Résultats}

\section{Données démographiques}

Les données démographiques n'ont pas été totalement collectées pour cinq étudiants : un étudiant du groupe A et trois étudiants du groupe $\mathrm{B}$ n'ont pas répondu au questionnaire, et un étudiant du groupe A a noté son année d'études à la place de son âge.

Le groupe A se composait de 42 étudiants âgés de $24,5 \pm 1,9$ ans en moyenne, avec $57,1 \%$ de femmes et $40,5 \%$ d'hommes (sex-ratio $=0,71$ ). Le groupe B se composait de 48 étudiants âgés de $24,8 \pm 2,6$ ans, avec $62,5 \%$ de femmes et $31,2 \%$ d'hommes (sex-ratio $=0,50)$ (Tableau I). Les deux groupes ne différaient pas statistiquement pour l'âge $(\mathrm{t}(83)=0,65 ; p=0,52)$ ou le sexe $\left(\chi^{2}(1)=0,31 ; p=0,58\right)$.

\section{Sites Internet consultés}

Pour répondre au cas clinique, 91 sites ont été consultés par les étudiants. Les sites les plus consultés ont été Wikipédia ( $81 \%$ de consultation dans le groupe A, $67 \%$ dans le groupe B), Planetoscope.com $(57 \%$ et $46 \%)$, Doctissimo (31\% et $19 \%)$, le site de la Sécurité sociale Ameli.fr (29\% et $38 \%$ ), le site de l'Organisation mondiale de la santé Who.int (21\% et $35 \%$ ) et le site de la Société nationale française de gastro-entérologie Snfge.org (19\% et $27 \%$ ) (Tableau II).

La consultation du site de la Haute autorité de santé (HAS) différait statistiquement entre les groupes avec $7 \%$ des étudiants du groupe A et $23 \%$ du groupe $\mathrm{B}(p=0,04)$. On note une tendance à une consultation plus fréquente du site de l'Organisation mondiale de la santé (OMS) Who.int dans le groupe B ( $35 \%$ versus $21 \%$ dans le groupe A, $p=0,17$ ), et une consultation moins fréquente de Wikipédia ( $67 \%$ contre $81 \%, p=0,15)$ et Doctissimo (19\% contre $31 \%, p=0,22)$.

Google était le moteur de recherche le plus fréquemment consulté dans les deux groupes et significativement plus consulté dans le groupe B (100\% de consultation) que dans le groupe A $(90 \%, p=0,04)$. Les autres moteurs de 


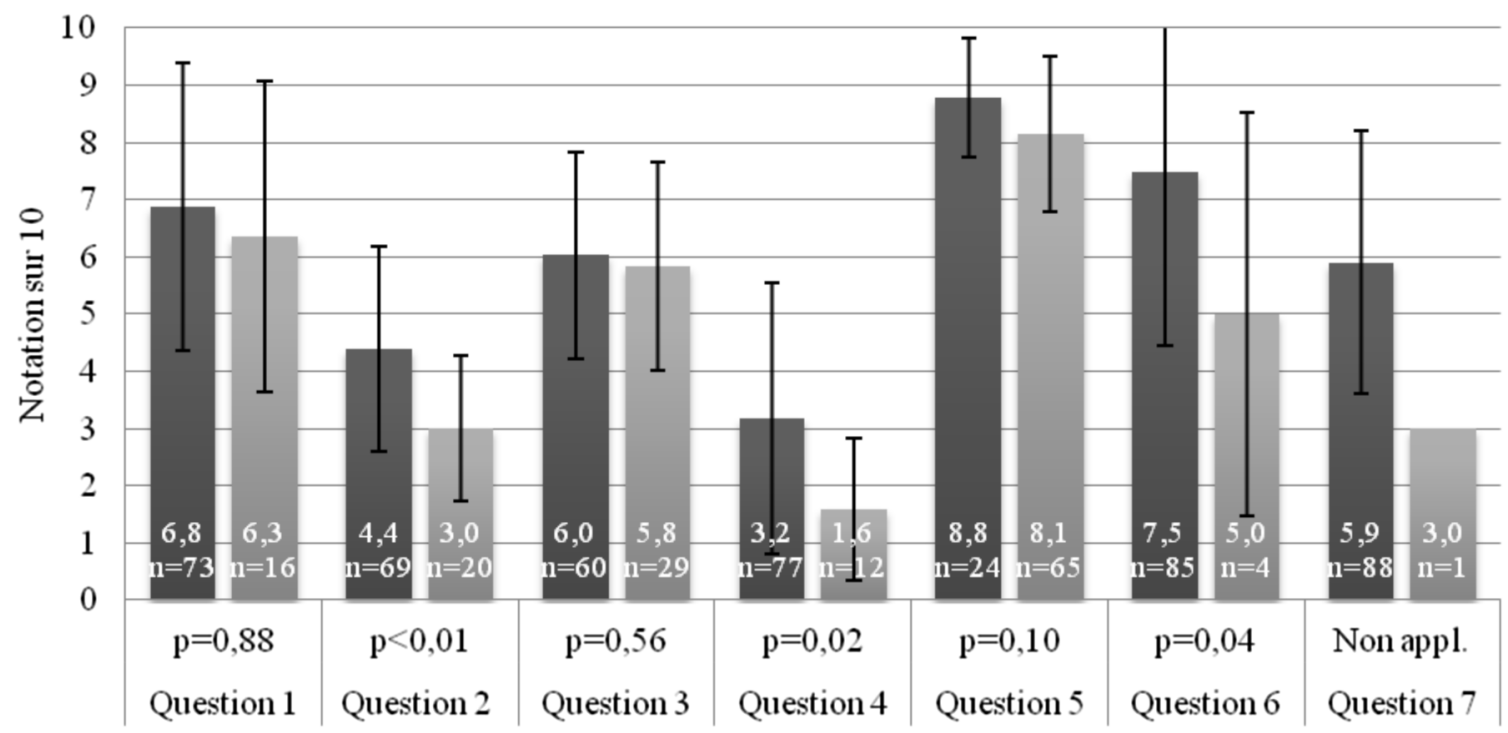

\section{- Groupe ay ant consulté un moteur de recherche $\quad$ Groupen'ay ant pas consulté de moteur de recherche}

Figure 1. Scores obtenus aux questions médicales selon la consultation d'Internet ou non. Question 1: quel est le traitement des hémorroïdes? Question 2: quels sont les symptômes d'un syndrome de sevrage tabagique? Question 3 : quels en sont les traitements possibles? Question 4: quelle est la définition du syndrome de Diogène? Question 5: citez dix moyens de contraception possibles. Question 6 : combien y a-t-il d'envenimations et de décès par morsure de serpent dans le monde chaque année? Question 7 : quelles sont les recommandations à donner en cas de morsure par un serpent?

Tableau III. Scores obtenus aux questions médicales dans les deux groupes d'étudiants.

\begin{tabular}{llll}
\hline & $\begin{array}{l}\text { Groupe A } \\
(n=42)\end{array}$ & $\begin{array}{l}\text { Groupe B } \\
(n=48)\end{array}$ & $\begin{array}{l}\text { Degré de } \\
\text { significativité }\end{array}$ \\
\hline Question 1 & 6,79 & 6,76 & $\mathrm{t}(88)=0,1 ; p=0,95$ \\
Question 2 & 4,14 & 4,00 & $\mathrm{t}(88)=0,4 ; p=0,66$ \\
Question 3 & 5,90 & 6,00 & $\mathrm{t}(88)=-0,2 ; p=0,85$ \\
Question 4 & 3,38 & 2,67 & $\mathrm{t}(88)=1,3 ; p=0,19$ \\
Question 5 & 8,31 & 8,31 & $\mathrm{t}(88)=0,0 ; p=0,99$ \\
Question 6 & 7,38 & 7,29 & $\mathrm{t}(88)=0,1 ; p=0,89$ \\
Question 7 & 5,93 & 5,85 & $\mathrm{t}(88)=0,2 ; p=0,88$ \\
\hline
\end{tabular}

Les résultats sont exprimés en score sur 10 points. Question 1 : quel est le traitement des hémorrö̈des? Question 2: quels sont les symptômes d'un syndrome de sevrage tabagique? Question 3: quels en sont les traitements possibles? Question 4: quelle est la définition du syndrome de Diogène? Question 5 : citez dix moyens de contraception possibles. Question 6 : combien y a-t-il d'envenimations et de décès par morsure de serpent dans le monde chaque année? Question 7 : quelles sont les recommandations à donner en cas de morsure par un serpent?

recherche utilisés ont été le moteur de recherche généraliste Yahoo (4\% dans le groupe $\mathrm{A}$ et $7 \%$ dans le groupe $\mathrm{B}, p=0,66)$, le moteur de recherche dérivé Scholar. google.fr $(0 \%$ dans le groupe A, $12 \%$ dans le groupe B, $p=0,06)$ et le moteur de recherche spécialisé de l'OMS (0\% dans le groupe A, $2 \%$ dans le groupe B, $p=1$ ).

Le site de la HAS a été significativement plus consulté pour la question 3 (quel est le traitement d'un syndrome de sevrage tabagique?) dans le groupe B avec $17 \%$ de consultation contre $2 \%$ dans le groupe A (test exact de
Fisher : $p=0,03 ;$ IC $95 \%[1,0 ; 371,9]$, $\mathrm{OR}=8,1)$. Aucune autre différence significative de consultation de site Internet ou de moteur de recherche n'a été retrouvée pour les autres questions.

\section{Scores d'évaluation obtenus au cas clinique}

Il n'y avait pas de différence significative dans les scores obtenus à chaque question entre les groupes A et B (Tableau III).

Les scores obtenus par les étudiants ont aussi été analysés en fonction de la consultation d'un moteur de recherche ou non. Nous avons ainsi réparti les 90 étudiants en un premier groupe ayant consulté un moteur de recherche pour répondre à la question analysée et un deuxième groupe ayant répondu sans l'aide d'Internet. Une différence significative des scores obtenus aux questions 2,4 et 6 a été retrouvée entre les participants ayant consulté un moteur de recherche et ceux ne l'ayant pas fait (Figure 1). À la question 2 (quels sont les symptômes d'un syndrome de sevrage tabagique?), les étudiants ayant consulté un moteur de recherche ont obtenu une moyenne de $4,4 / 10$ contre $3,0 / 10$ pour ceux n'en ayant pas consulté $(\mathrm{t}(87)=3,2 ; p<0,01)$. À la question 4 (quelle est la définition du syndrome de Diogène?), on retrouvait respectivement des moyennes de $3,2 / 10$ contre $1,6 / 10(p=0,02)$ et à la question 6 (combien y a-t-il d'envenimations et de décès par morsure de serpent dans le monde chaque année?) respectivement $7,5 / 10$ et $5,0 / 10(p=0,04)$.

Nous avons ensuite réparti les scores des étudiants en fonction des sites qu'ils avaient consultés pour répondre à la question correspondante (Tableau IV). Les étudiants 
C. Zumstein et al.

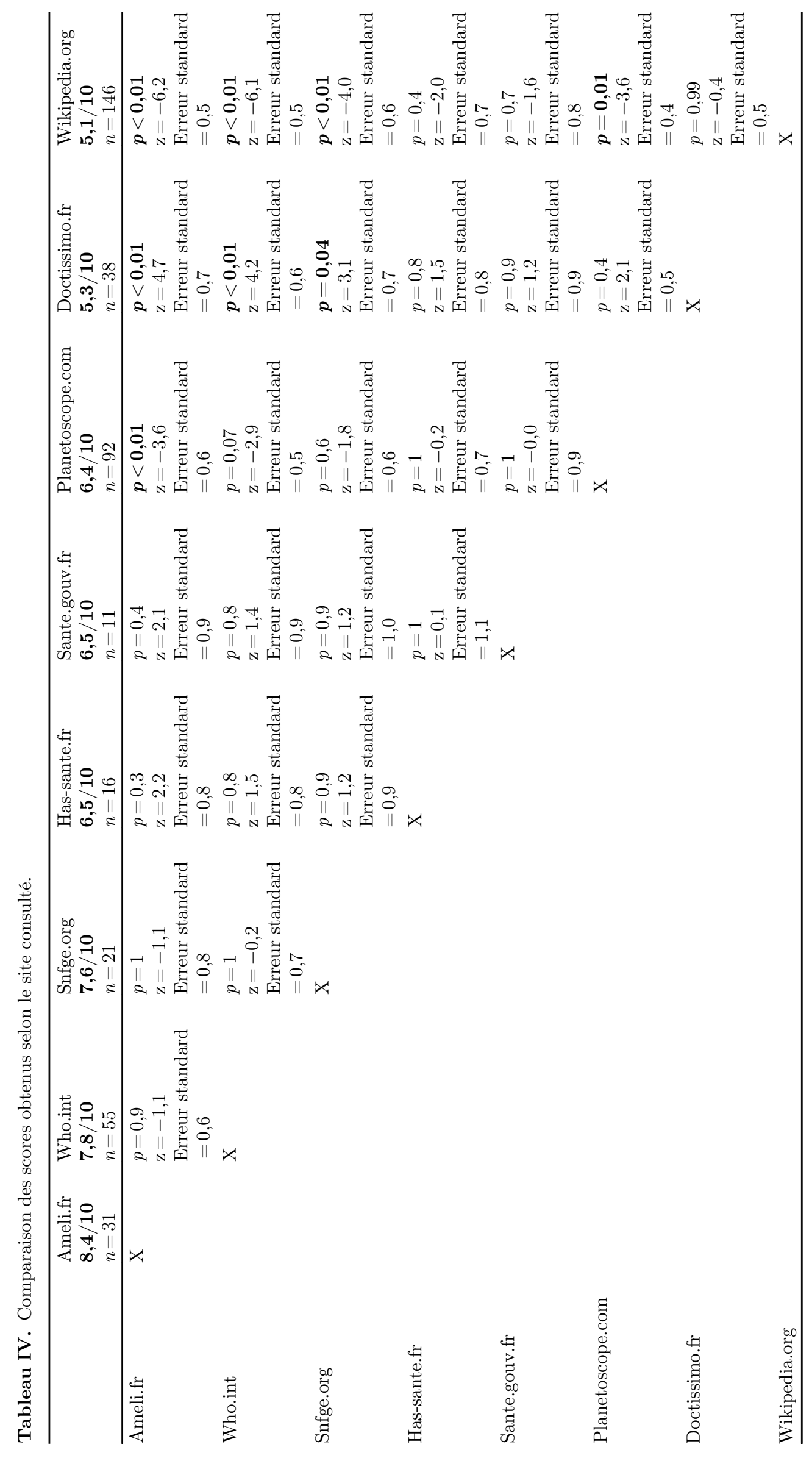


ayant consulté Wikipédia et Doctissimo ont obtenu un score moyen respectif de 5,1 et $5,3 / 10$, moyennes significativement plus basses que ceux ayant utilisé les sites Ameli.fr $(8,4 / 10, p<0,01)$, le site de l'OMS (7,8/10, $p<0,01)$ et le site de la Société nationale française de gastro-entérologie $(7,6 / 10$, respectivement $p<0,01$ et $p=0,04)$. La moyenne obtenue en utilisant Wikipédia était significativement plus basse que celle obtenue en consultant Planetoscope.com $(6,4 / 10 ; p=0,01)$, elle-même inférieure à celle obtenue avec le site Ameli.fr $(p=0,01)$. Aucune différence significative n'a été retrouvée en comparaison avec le site de la HAS ou Sante.gouv. fr. Les scores obtenus par les étudiants ayant utilisé Doctissimo et ceux ayant utilisé Wikipédia n'étaient pas statistiquement différents $(p=0,99)$.

\section{Techniques de recherche utilisées pour répondre au cas clinique}

Il n'y avait pas de différence significative de nombre d'URL consultées entre les groupes A et B (respectivement $23,2 \pm 9,0$ et $28,3 \pm 12,6 ; p=0,08 ; n=89$ ), mais le groupe $\mathrm{B}$ a consulté significativement plus de domaines que le groupe A $(6,8 \pm 1,6$ versus $6,0 \pm 2,1 ; p=0,03 ; n=89)$. Pour la question 7 (quelles sont les recommandations à donner en cas de morsure par un serpent?), le groupe B a consulté significativement plus de domaines que le groupe A (en moyenne 2,2 $\pm 1,2$ domaines contre 1,7 $\pm 0,8$; $p=0,04 ; n=90)$. Aucune autre différence significative de nombre de domaines ou d'URL consultés n'a été retrouvée entre les groupes pour les autres questions.

Aucune différence significative n'a été observée entre les deux groupes concernant le temps de réponse global aux questions de l'épreuve (respectivement 27 minutes 30 secondes contre 29 minutes 25 secondes, $p=0,15$; $n=88$ ) et le temps moyen passé par URL (respectivement 1 minute 21 secondes contre 1 minute 13 secondes, $p=0,25 ; n=87)$.

Aucun mot de liaison n'a été utilisé pour affiner les recherches : les mots-clés ont été juxtaposés dans la barre de recherche. Un seul étudiant a utilisé les guillemets pour séparer ses mots-clés. Toutes les pages consultées étaient en langue française, sauf pour quatre participants qui ont consulté une à deux pages en anglais.

Respectivement, $57 \%$ et $46 \%$ d'étudiants des groupes $\mathrm{A}$ et $\mathrm{B}$ ont procédé à un copier-coller pour donner la définition du syndrome de Diogène $(p=0,39 ; n=90)$ en question 4.

Respectivement, $14 \%$ et $13 \%$ des étudiants de chaque groupe ont cité moins de dix moyens de contraception parmi les dix demandés en question 5 sans faire de recherche sur Internet $(p=1 ; n=90)$.

Respectivement, $40 \%$ et $35 \%$ des étudiants des groupes A et B ont confondu les mots «morsure» et «envenimation» qui figuraient dans l'énoncé de la question 6 (combien y a-t-il d'envenimations et de décès par morsure de serpent dans le monde chaque année?) $(p=0,78 ; n=90)$ alors que souvent le site Internet consulté expliquait cette différence.

\section{Résultats du questionnaire de sensibilisation}

Les réponses des étudiants au questionnaire de sensibilisation ont été réunies en un groupe unique afin de réaliser une analyse globale. Les étudiants ont déclaré consulter en majorité les sites des collèges nationaux des enseignants $(98,8 \%)$, le site de la HAS $(94,2 \%)$ et le site Wikipédia $(88,4 \%)$ (Figure 2). D'autres sites sont consultés significativement moins souvent, comme les sites des sociétés savantes (59,3\%), les revues médicales en ligne (54,7\%), le site Pubmed (48,8\%) et Doctissimo (38,4\%). Une proportion similaire d'étudiants déclare consulter d'autres sites que ceux proposés dans notre questionnaire (39,5\%). Trois sites proposés ne sont que très peu consultés, $15,1 \%$ des étudiants déclarant consulter Bmlweb.org, 7,0\% Aufeminin.com et seulement 3,5\% la base de données Cochrane.

Avec des moyennes respectives de 9,5/10 et 9,4/10, le site de la HAS et les sites des collèges nationaux des enseignants sont les plus fiables selon les étudiants (Figure 3). Viennent ensuite les sites des sociétés savantes (9,0/10), Pubmed (8,9/ $10)$, la base de données Cochrane $(8,2 / 10)$, les sites des revues médicales en ligne $(8,1 / 10)$, Bmlweb.org $(7,2 / 10)$ et Wikipédia $(5,7 / 10)$. Ces sites sont jugés plus fiables que Doctissimo (3,2/10) et Aufeminin.com (2,2/10).

Les étudiants en médecine ont affirmé consulter en moyenne 2,7 sites Internet lors d'une même recherche scientifique ou médicale (IC 95\% : [2,6-2,9]) (écart-type $=$ $0,77)$.

Seuls deux étudiants ont affirmé connaître le logo HONcode $(2,3 \%$ (IC $95 \%$ : [0,3-8,2])) ; un seul pouvait en donner la signification. Nous avons observé une proportion de 22,1\% (IC $95 \%$ : [13,9-32,3\%]) d'étudiants utilisant leur ENT pour accéder à la documentation en ligne de l'Université de Strasbourg.

Les hommes ont significativement plus souvent déclaré consulter Pubmed que les femmes avec un pourcentage de consultation de $69 \%$ contre $37 \% \quad(p=0,01 ; n=86)$. Wikipédia est jugé significativement plus fiable par les hommes que par les femmes $(6,2 / 10$ contre $5,4 / 10, p=0,02$; $n=86)$ sans autre différence de fiabilité entre hommes et femmes.

\section{Discussion}

\section{Pratiques de recherche sans sensibilisation}

Pour répondre à des questions introduites par un cas clinique de médecine générale, les étudiants consultent en majorité des sites destinés au grand public au détriment de sites médicaux de référence et institutionnels. Si certaines de leurs déclarations dans le questionnaire coïncident avec les données de la mise en situation, d'autres s'en éloignent sensiblement. Leurs déclarations diffèrent de celles obtenues par Ricard et al. [4]: en 2005, les étudiants déclaraient rarement fréquenter les sites institutionnels alors qu'ils sont rapidement cités dans notre étude. Dans notre étude comme dans celle de Egle et al. [9] en 2015, le site «grand public» Wikipédia était souvent cité $(7 \%$, $n=73)$ tout comme Pubmed ( $4 \%, n=73)$. 


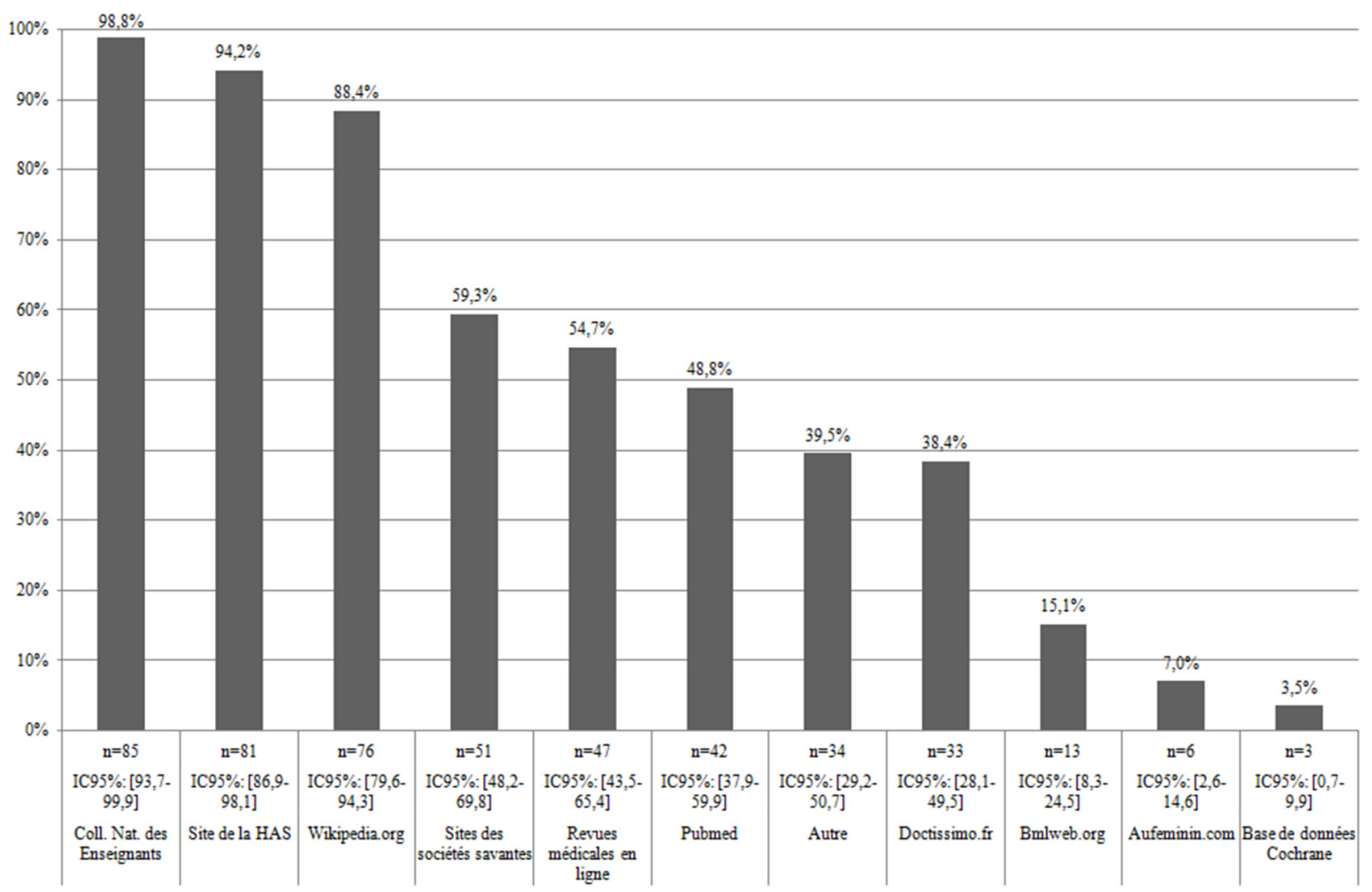

Figure 2. Sites consultés par les étudiants selon leurs déclarations au questionnaire. Coll. Nat. des Enseignants : Collèges nationaux des enseignants, HAS : Haute Autorité de santé.

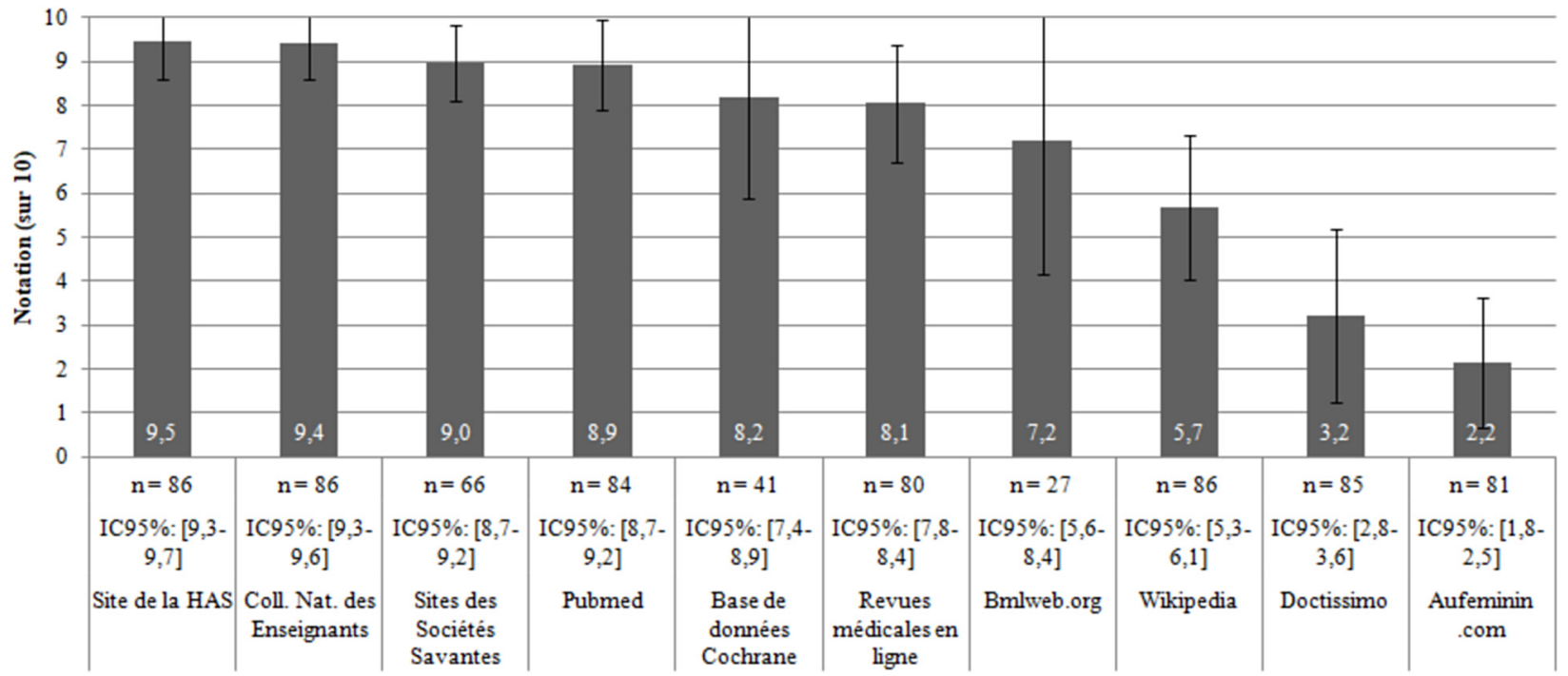

Figure 3. Notation des sites Internet selon les déclarations des étudiants. Coll. Nat. des Enseignants: Collèges nationaux des enseignants, HAS : Haute Autorité de santé.

Ces résultats mettent à jour un biais de désirabilité sociale lors des réponses des étudiants au questionnaire de sensibilisation: les discordances observées entre déclarations des étudiants et observation en situation peuvent s'expliquer par une tendance des étudiants à répondre d'une façon socialement acceptable au détriment de leurs convictions propres, ce qui peut diminuer le degré de validité de leurs déclarations. Ce biais avait aussi été observé dans l'étude d'Eysenbach et al. [15].

Nos résultats permettent également de nous interroger sur les facteurs qui expliqueraient la consultation principale de sites «grand public». Des entretiens avec les 
étudiants permettraient de clarifier les motivations soustendant leurs habitudes de recherche sur Internet.

Les étudiants estiment pourtant que les sites de référence sont plus fiables que les sites «grand public», sentiment confirmé par les notes obtenues lors de la mise en situation. Cependant, la littérature existante ne permet pas de conclure quant à la fiabilité des sites de santé. Certaines études $[1,2,6,7,9]$ confirment nos données en jugeant les sites professionnels plus fiables que les sites «grand public», mais d'autres $[3,8,12-14]$ sont en désaccord et placent Wikipédia parmi les sites jugés les plus fiables au même niveau que l'US National Cancer Institute par exemple.

L'outil d'évaluation des sites de santé utilisé diffère d'une étude à l'autre. Notre analyse de la fiabilité des sites s'est basée sur les déclarations arbitraires des étudiants, ainsi que sur les scores obtenus par les étudiants aux différentes questions du cas clinique en fonction des sites consultés. D'autres études ont utilisé le logo HONcode $[2,6]$, une évaluation subjective réalisée par des experts $[1,6,7,12,13]$ ou les outils DISCERN [3] ou LIDA [2]. Une évaluation plus pertinente de la qualité des sites devra reposer sur l'utilisation d'un outil standardisé et validé par la communauté scientifique.

\section{Sensibilisation des étudiants}

L'utilisation d'un questionnaire interrogeant les étudiants sur leurs pratiques de recherche sur Internet a permis d'augmenter significativement la fréquence de consultation du site de la HAS et d'observer une tendance à une plus forte consultation du site de l'OMS, et une moindre consultation de Doctissimo et Wikipédia, deux sites «grand public» n'affichant pas le HONcode. La sensibilisation semble donc avoir initié une réflexion des étudiants sur leurs pratiques de recherche sur Internet puisqu'ils ont consulté des sites plus fiables lorsque le questionnaire précédait leur recherche. Il faut souligner que le questionnaire n'apportait aucun conseil de recherche, mais amenait les étudiants à réfléchir sur leurs pratiques en les interrogeant sur leurs habitudes et leurs connaissances du domaine. Si leurs critères de jugement n'ont pas été directement évalués, un des étudiants du groupe B a cliqué sur l'onglet «qui sommes-nous? » d'un site pour juger des qualifications des auteurs. Ainsi, le simple fait de poser des questions réflexives sur les pratiques de recherche semble améliorer la qualité de ces pratiques. Certains critères d'évaluation des sites médicaux ont été évoqués lors de groupes de discussion focalisés réalisés par Eysenbach et al. [15] et peuvent être des pistes pour affiner les questions posées : évaluez-vous l'auteur et ses qualifications, favorisez-vous la consultation de sites institutionnels, vérifiez-vous la date de rédaction et de mise à jour, vérifiez-vous la présence de références et la validation du site par une autorité compétente?

Les étudiants sensibilisés ont aussi consulté plus de domaines que les autres et ont ainsi multiplié leurs sources d'information pour les recouper et s'assurer de la pertinence d'informations similaires sur plusieurs sites.
L'ajout d'une question concernant la multiplication des sources pourrait aussi favoriser cette pratique qui permet une recherche plus fiable.

Le questionnaire de sensibilisation n'a cependant pas permis d'améliorer la qualité des réponses des étudiants au cas clinique, mais nous avons observé pour trois questions une augmentation significative des scores des étudiants ayant utilisé Internet par rapport à ceux qui se sont seulement référés à leurs propres connaissances. Internet peut donc permettre de compléter efficacement les connaissances des étudiants et d'améliorer la qualité de leurs réponses, d'où l'importance de savoir utiliser cet outil efficacement et de savoir évaluer la fiabilité des sites consultés.

\section{Techniques de recherche}

Peu d'étudiants ont utilisé un autre moteur de recherche que Google.fr, résultat en accord avec ceux de l'étude de Ricard et al. [4]. Cette pratique semble justifiée puisque dans une étude menée par Ilic et al. [3], Google.fr était considéré comme le moteur de recherche le plus efficace en comparaison aux autres moteurs de recherche.

Aucun étudiant n'a utilisé de mots de liaison entre les mots-clés ou de filtres de recherche avancés, ce qui est concordant avec les résultats de l'étude d'Eysenbach et al. [15].

La littérature ne permet pas de conclure quant à la fréquence de consultation de sites en anglais: aucun participant $(n=16)$ de l'étude d'Eysenbach et al. [15] n'avait consulté de site en langue étrangère ; très peu l'ont fait dans notre étude, contrairement aux $21 \%(n=143)$ et $54 \%(n=71)$ des étudiants respectivement en $3^{\mathrm{e}}$ et 6 année de médecine inclus par Hattab et al. [5] mais dont les résultats ne se basaient que sur les déclarations de ces étudiants, avec un possible biais de désirabilité sociale. Or se limiter à des recherches en français peut diminuer la pertinence des résultats, la littérature scientifique étant surtout publiée en anglais.

Eysenbach et al. [15] trouvaient une moyenne de temps passé par page consultée (URL) similaire à la nôtre; une minute semble donc suffire à déterminer la fiabilité d'une page Internet et son utilité pour obtenir les réponses voulues. Le logo HONcode est peu connu des étudiants mais, suite à notre étude, deux étudiants ont cherché sa signification sur nos ordinateurs.

La moitié des étudiants ont copié-collé les données du site consulté pour définir le syndrome de Diogène, sans tenter de se les approprier pour répondre aux questions annexées au cas clinique. Le pourcentage d'étudiants ayant fait ce «plagiat» sans citer les sources n'a pas été amélioré par l'action de sensibilisation.

Plus du tiers des étudiants ont confondu les termes «morsure » et «envenimation» dans leur réponse, alors que les trois sites les plus consultés pour répondre expliquent la différence entre ces termes. Beaucoup d'étudiants ne prennent ainsi pas le temps de lire les quelques lignes d'un article mais se contentent de citer la première valeur chiffrée qu'ils trouvent. 


\section{Limites de l'étude}

Nous avons observé une différence significative de consultation du site de la HAS entre les groupes, mais certaines tendances n'ont pas pu être confirmées statistiquement pour des raisons techniques nous empêchant d'inclure plus de sujets. Il nous aurait fallu inclure 161 étudiants dans chaque groupe pour avoir $80 \%$ de chances d'observer d'autres différences significatives.

Par ailleurs, bien qu'introduites par un cas clinique, les questions visant à évaluer les connaissances des étudiants étaient totalement décontextualisées; elles auraient en effet pu être posées indépendamment de la vignette clinique puisqu'elles n'impliquaient pas que les étudiants examinent de façon critique la façon d'appliquer de façon pertinente l'information concernée à la spécificité du cas. Aucune conclusion ne peut donc être tirée quant à la transférabilité des apprentissages effectués dans le cadre de l'étude.

Notre choix de nous intéresser à des étudiants en sixième et dernière année du cursus pré-gradué des études de médecine peut sembler étonnant alors que l'on considère qu'un apprentissage doit survenir précocement dans un cursus pour aboutir à un changement. Cependant, les étudiants d'années inférieures étant supervisés, ils n'ont pas encore été confrontés à des prises de décision pour leurs patients et n'ont donc pas encore expérimenté la consultation d'Internet dans le cadre d'une consultation médicale.

Enfin, notre étude a évalué directement l'impact de la sensibilisation, sans que l'on puisse préjuger de la durabilité de ces effets, qui seraient à réévaluer à distance de notre action de sensibilisation.

\section{Conclusion}

Confrontés dans leur pratique professionnelle à Internet, les étudiants reconnaissent manquer de ressources méthodologiques en la matière. Notre étude nous a permis d'observer leurs pratiques et d'évaluer l'impact d'une sensibilisation par un questionnaire les interrogeant sur ces pratiques. Les étudiants en médecine consultent majoritairement des sites destinés au grand public, comme Wikipédia ou Doctissimo, alors qu'ils les jugent significativement moins fiables que des sites médicaux de référence comme le site de la HAS. L'utilisation d'un questionnaire leur permettant de s'interroger sur leurs pratiques semble favoriser une utilisation d'Internet plus efficiente. En effet, les étudiants sensibilisés utilisent plus fréquemment des sites médicaux de référence et multiplient le nombre de sources d'information médicale consultées. Ils recoupent ainsi les informations qu'ils lisent, ce qui rend leurs recherches plus pertinentes. La sensibilisation n'a pas permis d'améliorer les réponses des étudiants au cas clinique, mais les étudiants ayant utilisé Internet ont obtenu de meilleurs scores que ceux qui ont répondu sans avoir consulté Internet. Internet est donc un outil bénéfique pour les futurs médecins afin de compléter, mettre à jour ou stimuler leurs connaissances. L'apprentissage efficace de son utilisation est un enjeu important pour permettre une prise en charge médicale optimale des patients. Si des formations existent déjà, une réflexion supplémentaire est nécessaire afin d'adapter l'enseignement aux besoins et demandes des étudiants en médecine, en intégrant l'apprentissage d'une lecture critique et efficace des informations. Les futurs professionnels de santé doivent développer leur capacité réflexive grâce à une prise de conscience individuelle et collective de leurs lacunes en matière de recherche professionnelle sur Internet.

\section{Contributions}

Carine Zumstein a participé à la conception du protocole de recherche, au recueil des données, à l'analyse statistique, à l'interprétation des résultats et à l'écriture du manuscrit. Claire Kam a participé à la conception du protocole de recherche, à l'interprétation des résultats, à l'écriture du manuscrit. Pierrick Le Borgne et Pierre Vidailhet ont participé à l'interprétation des résultats et à l'écriture du manuscrit. François Lefebvre a participé à l'analyse statistique. Michel Lévêque a participé à l'écriture du manuscrit.

\section{Déclaration d'intérêts}

Les auteurs déclarent n'avoir aucun lien d'intérêt concernant les données publiées dans cet article.

\section{Valorisation académique et scientifique}

Ce travail a été présenté sous forme de communication affichée au $10^{\mathrm{e}}$ Congrès européen de médecine d'urgence (European Congress on Emergency Medicine) à Vienne (Autriche) en 2016.

Ce travail a fait l'objet du travail de thèse pour le Diplôme d'état de doctorat en médecine (qualification médecine générale) de Carine Zumstein.

\section{Approbation éthique}

Cette étude a été déclarée au registre des activités de traitements conformément aux dispositions réglementaires de la loi informatique et liberté et a obtenu l'avis favorable consultatif du comité d'éthique des facultés de médecine, d'odontologie, de pharmacie, des écoles d'infirmières, de kinésithérapie, de maïeutique et des Hôpitaux de Strasbourg (numéro d'enregistrement 2017-82).

\section{Remerciements}

Nous remercions le Dr Thierry Pelaccia pour sa relecture de l'article. 


\section{Références}

1. Mathur S, Shanti N, Brkaric M, Sood V, Kubeck J, Paulino $\mathrm{C}$, et al. Surfing for scoliosis: the quality of information available on the Internet. Spine 2005;30:2695-700.

2. Tavare AN, Alsafi A, Hamady MS. Analysis of the quality of information obtained about uterine artery embolization from the Internet. Cardiovasc Intervent Radiol 2012;35:1355-62.

3. Ilic D, Bessell TL, Silagy CA, Green S. Specialized medical search-engines are no better than general search-engines in sourcing consumer information about androgen deficiency. Hum Reprod 2003;18:557-61.

4. Ricard J-D., Lejoyeux M, El-Ghoneimi A, Matheron S, Maillard D, Crickx B, et al. Utilisation des nouvelles technologies de l'information et de la communication par les étudiants en médecine. Enquête de pratique et mise en situation. Pédagogie Médicale 2005;6:112-22.

5. Hattab NM, Lahmiti S, Ben Abdelaziz A, Saidi H, Fikry T. Internet and medical student in Marrakech Ann Afr Med 2010;9:68-72.

6. Meric F, Bernstam EV, Mirza NQ, Hunt KK, Ames FC, Ross MI, et al. Breast cancer on the world wide web: crosssectional survey of quality of information and popularity of websites. BMJ 2002;324:577-81.

7. Leithner A, Maurer-Ertl W, Glehr M, Friesenbichler J, Leithner K, Windhager R. Wikipedia and osteosarcoma: a trustworthy patients' information? J Am Med Inform Assoc 2010;17:373-74.
8. Sullivan TB, Anderson JT, Ahn UM, Ahn NU. Can Internet information on vertebroplasty be a reliable means of patient self-education? Clin Orthop 2014;472:1597-604.

9. Egle JP, Smeenge DM, Kassem KM, Mittal VK. The Internet school of medicine: use of electronic resources by medical trainees and the reliability of those resources. J Surg Educ 2015;72:316-20.

10. Pletneva N, Cruchet S, Simonet M-A, Kajiwara M, Boyer C. Évolution d'internet dans le domaine médical. 2010 [Online]. Disponible sur: http://www.hon.ch/Global/pdf/ 2010_Internet_use_Trends_f.pdf.

11. Murray E, Lo B, Pollack L, Donelan K, Catania J, Lee K, et al. The impact of health information on the Internet on health care and the physician-patient relationship: national U.S. survey among 1050 U.S. physicians. J Med Internet Res 2003;5:e17.

12. Wang L, Wang J, Wang M, Li Y, Liang Y, Xu D. Using internet search engines to obtain medical information: a comparative study. J Med Internet Res 2012;14:e74.

13. Lutsky K, Bernstein J, Beredjiklian P. Quality of information on the Internet about carpal tunnel syndrome: an update. Orthopedics 2013;36:e1038-041.

14. Bould MD, Hladkowicz ES, Pigford A-AE, Ufholz L-A., Postonogova T, Shin E, et al. References that anyone can edit: review of Wikipedia citations in peer reviewed health science literature. BMJ 2014;348:g1585.

15. Eysenbach G, Köhler C. How do consumers search for and appraise health information on the world wide web? Qualitative study using focus groups, usability tests, and in-depth interviews. BMJ 2002;324:573-77.

Citation de l'article : Zumstein C., Kam C., Borgne P.L., Lefebvre F., Leveque M., Vidailhet P., Étudiants en médecine et Internet : quelles pratiques de recherche et comment les améliorer? Pédagogie Médicale 2017:18;65-81 


\section{Annexe 1: Formulaire de consentement.}

Madame, Monsieur,

Nous vous proposons de participer à un travail de recherche pédagogique intitulé « Répondre à un cas clinique avec l'aide d'Internet ». Son but est d'évaluer votre façon de répondre à un cas clinique et la façon dont vous vous aidez d'Internet pour répondre à ce cas clinique.

Deux groupes d'étudiants en $2^{\mathrm{e}}$ cycle des études médicales vont être soumis à un questionnaire déclaratif (à remplir directement sur Internet sur cette page :

https://docs.google.com/forms/d/1-j1qT1hunDeB94yCU3NyJ8NzhCmn1x7iBE1LEMyheg/viewform) et à un cas clinique (réponses sur papier libre avec numéro d'anonymat) auquel ils devront répondre avec l'aide d'un accès Internet fourni par Mozilla Firefox et le moteur de recherche Google. L'un des deux groupes répondra d'abord au questionnaire déclaratif puis au cas clinique tandis que l'autre groupe répondra en premier au cas clinique, puis au questionnaire déclaratif.

Nous vous informons que :

- L'anonymat des réponses est garanti,

- Le secret de vos réponses est total,

- Il sera impossible d'identifier un quelconque participant,

- Le travail réalisé se fait sous le seul regard d'un contributeur de l'étude,

- Votre participation est entièrement indépendante de vos études et ne changera en rien

les conditions de votre stage,

- Votre consentement est révocable à tout moment,

- Ce travail a fait l'objet d'une déclaration à la CNIL.

Je soussigné(e) : (NOM Prénom)

Numéro d'anonymat :

Groupe débutant par :

Ai reçu toutes les informations nécessaires pour comprendre les principes et les modalités de l'étude « Répondre à un cas clinique avec l'aide d'Internet » réalisée à l'Unité de Simulation Pédagogique de Strasbourg durant l'année 2015. En conséquence :

Je donne mon accord pour y participer $\quad \square$ Je refuse d'y participer

Fait à Strasbourg, le : ____/2015

Signature : 


\section{Annexe 2: Cas clinique avec sa grille de correction.}

Un patient vous est adressé en service de Gérontologie pour des rectorragies. L'examen clinique retrouve des hémorroïdes. On note dans ses antécédents un adénocarcinome prostatique, une cholécystectomie, un glaucome, une HTA et un tabagisme actif.

\section{1) Quel est le traitement des hémorrö̈des ?}

sur 10

Règles hygiéno-diététiques / mesures associées contre constipation, laxatifs

Traitement médical local, per os

Traitement instrumental / endoscopique sclérose, coagulation, ligature

Traitement chirurgical thrombectomie, hémorroïdectomie, anopexie

\begin{tabular}{l|r|}
\cline { 2 - 2 } & $\begin{array}{c}\text { sur 10 } \\
\text { points }\end{array}$ \\
\hline si une proposition & 1,5 \\
si une proposition & 1 \\
\hline si une proposition & 1,5 \\
\hline si une proposition & 1,5 \\
\hline
\end{tabular}

2) Au cours de son hospitalisation, il présente un syndrome de sevrage suite à l'arrêt du tabac. Quels en sont les symptômes possibles?

sur 10

points

\begin{tabular}{|l|r|}
\hline Humeur dépressive & 1 \\
\hline Insomnie & 1 \\
\hline Irritabilité, frustration, colère & 1 \\
\hline Anxiété & 1 \\
\hline Difficulté de concentration & 1 \\
\hline Agitation & 1 \\
\hline Ralentissement du pouls & 1 \\
\hline Augmentation de l'appétit, prise de poids rapide & 1 \\
\hline $\begin{array}{l}\text { Ces signes causent une souffrance clinique +/- altération du fonctionnement } \\
\text { social ou familial }\end{array}$ & 1 \\
\hline Ces signes ne sont pas expliqués par une maladie mentale & 1 \\
\hline
\end{tabular}

3) Quels sont les traitements possibles ?

sur 10 points

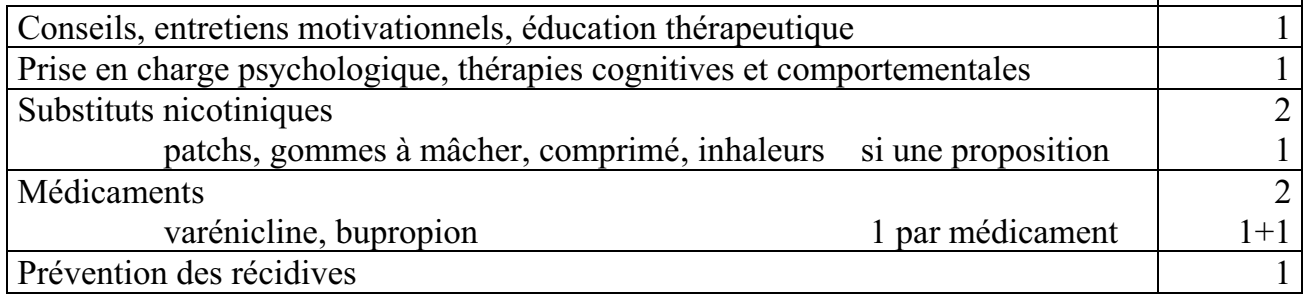


4) Le patient rentrera finalement à son domicile. En tant que médecin traitant, vous effectuez une visite de contrôle et vous évoquez un "syndrome de Diogène". Quelle en est la définition?

\begin{tabular}{|l|r|}
\hline Accumulation d'objets, syllogomanie & 1 \\
\hline Négligence de soi, incurie & 1 \\
\hline Absence de maladie psychiatrique & 1 \\
\hline Intelligence élevée, niveau d'éducation élevé, absence d'altération cognitive & 1 \\
\hline Isolement social & 1 \\
\hline Personnalité pré-morbide distante, méfiante, agressive, labilité émotionnelle & 1 \\
\hline Refus des aides, aides vécues comme intrusives & 1 \\
\hline Absence de honte, anosognosie & 1 \\
\hline Âge > 65 ans & 1 \\
\hline Désintérêt de l'environnement & 1 \\
\hline
\end{tabular}

5) Au moment de votre visite, sa fille qui est présente vous demande de la conseiller pour sa contraception. Citez 10 moyens de contraception possibles.

sur 10

points

Pilule, patch, implant, anneau vaginal, stérilet, préservatif masculin ou féminin, diaphragme, cape cervicale, spermicides, stérilisation, méthodes naturelles, progestatifs injectables, abstinence

6) Elle vous confie vouloir voyager en Afrique et redouter les morsures de serpent. Combien y a-t-il d'envenimations et de décès par morsure de serpent dans le monde chaque année ?

7) Quelles sont les recommandations à lui donner en cas de morsure par un serpent ?

\begin{tabular}{|lr|}
\hline Appel des secours (112) & 1 \\
\hline Désinfecter la plaie & 1 \\
\hline Ôter bagues/bracelets/tout objet potentiellement compressif & 1 \\
\hline Bandage peu serré, pas de garrot & 1 \\
\hline Surélever le membre, immobiliser & 1 \\
\hline Rassurer, calmer la victime & 1 \\
\hline Ne pas sucer, cautériser, inciser & 1 \\
\hline Antalgiques simples, contre-indication des AINS et aspirine & 1 \\
\hline Pas d'aspivenin & 1 \\
\hline Mémoriser l'aspect du serpent & 1 \\
\hline
\end{tabular}




\section{Annexe 3: Questionnaire de sensibilisation.}

Quel est votre numéro d'anonymat ? *

En quelle année d'études êtes-vous ? *
D2N
D2R
D3N
D3R
D4N

$\square$ D4R

Quel âge avez-vous ?*

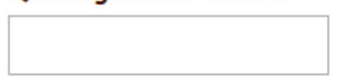

Quel est votre sexe ?*

Féminin

Masculin

Avez-vous consulté ces sites dans les 6 derniers mois pour une question médicale ou scientifique ?*

\begin{tabular}{lcc} 
& Oui & Non \\
\hline Wikipédia & 0 & 0 \\
\hline Doctissimo & 0 & 0 \\
\hline Aufeminin.com & 0 & 0 \\
\hline Bmlweb.org & 0 & 0 \\
\hline Pubmed & 0 & 0 \\
\hline Base de données Cochrane & 0 & 0 \\
\hline Revues médicales en ligne & 0 & 0 \\
\hline Site de la HAS & 0 & 0 \\
\hline Sites des Collèges Nationaux & 0 & 0 \\
\hline des Enseignants & 0 & 0 \\
\hline Sites des Sociétés Savantes & & \\
\hline
\end{tabular}


Avez-vous consulté un autre site ou une autre base de données en ligne dans les 6 derniers mois pour une question médicale ou scientifique ?*

$\square$ Oui

$\square$ Non

Si oui, la ou lesquelles?

Selon vous, quelle est la fiabilité des informations disponibles sur ces sites ? Notez-les de 1 (non fiable) à 10 (totalement fiable) *

\begin{tabular}{|c|c|c|c|c|c|c|c|c|c|c|c|}
\hline & 1 & 2 & 3 & 4 & 5 & 6 & 7 & 8 & 9 & 10 & $\begin{array}{l}\text { Je ne } \\
\text { connais } \\
\text { pas ce } \\
\text { site }\end{array}$ \\
\hline Wikipédia & 0 & $\bigcirc$ & 0 & 0 & $\mathrm{O}$ & $\mathrm{O}$ & 0 & 0 & 0 & 0 & 0 \\
\hline Doctissimo & 0 & $\bigcirc$ & 0 & 0 & 0 & 0 & $\mathrm{O}$ & 0 & $\bigcirc$ & 0 & 0 \\
\hline Aufeminin.com & $\bigcirc$ & $\bigcirc$ & 0 & 0 & 0 & 0 & 0 & 0 & $\bigcirc$ & 0 & 0 \\
\hline Bmlweb.org & 0 & 0 & 0 & 0 & $\bigcirc$ & 0 & $\bigcirc$ & $\bigcirc$ & $\bigcirc$ & $\bigcirc$ & $\bigcirc$ \\
\hline Pubmed & 0 & 0 & 0 & 0 & 0 & 0 & 0 & 0 & 0 & 0 & $\bigcirc$ \\
\hline $\begin{array}{l}\text { Base de } \\
\text { données } \\
\text { Cochrane }\end{array}$ & 0 & 0 & 0 & 0 & 0 & 0 & $\mathrm{O}$ & 0 & 0 & 0 & 0 \\
\hline $\begin{array}{l}\text { Revues } \\
\text { médicales en } \\
\text { ligne }\end{array}$ & 0 & 0 & 0 & 0 & 0 & 0 & 0 & 0 & 0 & 0 & $\mathrm{O}$ \\
\hline Site de la HAS & $\bigcirc$ & 0 & 0 & 0 & 0 & 0 & 0 & 0 & 0 & 0 & 0 \\
\hline $\begin{array}{l}\text { Sites des } \\
\text { Collèges } \\
\text { Nationaux des } \\
\text { Enseignants }\end{array}$ & 0 & 0 & 0 & 0 & 0 & 0 & 0 & 0 & 0 & 0 & 0 \\
\hline $\begin{array}{l}\text { Sites des } \\
\text { Sociétés } \\
\text { Savantes }\end{array}$ & 0 & 0 & 0 & 0 & 0 & 0 & 0 & 0 & 0 & $\mathrm{O}$ & 0 \\
\hline
\end{tabular}

Avez-vous utilisé votre E.N.T. dans les 6 derniers mois pour accéder à la documentation en ligne de l'Université ? *

Oui

Non 
En moyenne, combien de sites différents consultez-vous pour une même question médicale ou scientifique ?*

$\begin{array}{llllllllll}1 & 2 & 3 & 4 & 5 & 6 & 7 & 8 & 9 & 10\end{array}$

0000000000

Avez-vous déjà entendu parler du logo HONcode ? *

Oui

Non

Si oui, donnez-en la signification en quelques mots :

Votre réponse a bien été enregistrée. Merci de votre participation. 